\title{
Traumatismes Fermés du Cadre Duodeno- Pancréatique Observés au Centre Hospitalier Universitaire Sylvanus Olympio de Lomé (Togo) en Situation d'urgence: A Propos de Deux Cas
}

\author{
Kodjo Abossisso Sakiye, \\ Biréga Koutora, \\ Fousséni Alassani, \\ Kokou K. Kanassoua, \\ Badjona Songne-Gnamkoulamba,
}

Service des Urgences Chirurgicales, Centre Hospitalier Universitaire Sylvanus Olympio, Département de Chirurgie et Spécialités Chirurgicales, Faculté des Sciences de la Santé, Université de Lomé, Togo

\section{Résumé}

Objectif : Souligner les difficultés diagnostiques et thérapeutiques que posent les lésions traumatiques du cadre duodéno-pancréatique dans notre contexte d'urgence chirurgicale au CHU sylvanus Olympio (CHU SO) avec un plateau technique d'exploration paraclinique insuffisant. Méthodologie : Nous rapportons deux cas incluant les patients pris en charge pour des lésions traumatiques du cadre duodéno-pancréatique traitées chirurgicalement survenues dans les circonstances d'accidents de la voie publique, d'Août 2018 à Juillet 2019. Résultats : Nous rapportons 2 cas qui touchent surtout les sujets jeunes de sexe masculin et féminin âgés respectivement de 27 et 18 ans. Ces patients avaient été victime tous d'un accident de la voie publique et trois jours après avaient présenté un abdomen chirurgical aigu. En peropératoire, les lésions observées consistaient à des contusions du parenchyme pancréatique sans fracture et à une perforation duodénal D4 associée dans un cas. Aucun bilan morphologique ne permettait d'objecter une atteinte du canal de Wirsung. Dans tous les cas le traitement conservateur était privilégié avec une splénectomie réalisée dans un cas. Le pronostic était bon dans tous les cas. Conclusion : les lésions traumatiques du cadre duodéno-pancréatique sont rares et les accidents de la voie publique constituent la première cause. Elles s'observent surtout dans le cadre d'un polytraumatisme et le diagnostique en situation d'urgence au CHU SO se heurte une insuffisance des moyens d'exploration. 
Mots clés : Traumatisme, Duodénum, Pancréas

\title{
Closed Trauma of the Duodeno-Pancreatic Framework Observed at the University Hospital Sylvanus Olympio of Lomé (Togo) in Emergency Situation: About Two Cases
}

\author{
Kodjo Abossisso Sakiye, \\ Biréga Koutora, \\ Fousséni Alassani, \\ Kokou K. Kanassoua, \\ Badjona Songne-Gnamkoulamba,
}

Service des Urgences Chirurgicales, Centre Hospitalier Universitaire Sylvanus Olympio, Département de Chirurgie et Spécialités Chirurgicales, Faculté des Sciences de la Santé, Université de Lomé, Togo

\begin{abstract}
Objective: To underline the diagnostic and therapeutic difficulties posed by traumatic lesions of the duodeno-pancreatic framework in our context of surgical emergency at CHU SO with an insufficient technical paraclinical exploration platform. Methodology: we report two cases of including patients treated for traumatic duodeno-pancreatic injuries treated surgically in the circumstances of road accidents, from August 2018 to July 2019. Results: We report 2 cases that mainly affect young men and women aged 27 and 18 respectively. These patients had all been involved in a road accident and three days later had an acute surgical abdomen. Intraoperative lesions were pancreatic parenchymal bruising without fracture and duodenal D4 perforation associated with one case. No morphological assessment allowed one to object to an attack on the Wirsung Canal. In all cases conservative treatment was favored with a splenectomy performed in one case. The prognosis was good in all cases. Conclusion: traumatic lesions of the duodeno-pancreatic framework are rare and road accidents are the first cause. They are observed mainly in the context of a polytrauma and the diagnosis in emergency situation at the CHU SO faces an insufficiency of the means of exploration.
\end{abstract}

Keywords: Trauma, Duodenum, Pancreas 


\section{Introduction}

Les traumatismes fermés du bloc duodéno-pancréatique sont rares en raison de la position anatomique de ces organes en rétropéritoine profond qui leur assure une certaine protection (Farrell, 1996; Beyrouti, 2005). Ils s'observent surtout dans le cadre d'un polytraumatisme. Et du fait de la violence du traumatisme nécessaire pour provoquer une lésion de ce bloc, il s'y associe au moins une lésion d'un autre organe dans 90\% chez l'adulte (Feliciano, 1987). L'expression clinique initiale varie de l'absence de tout signe clinique initial à un tableau péritonéal franc; et dans le cas d'une atteinte pancréatique malheureusement il n'est pas possible de préjuger d'une atteinte canalaire en fonction de la symptomatologie (Bradley, 1998). Ils sont grevés d'une lourde morbi-mortalité pouvant atteindre 39\% surtout en cas de retard diagnostic (Jurkovich, 2013). De plus, la diversité et la complexité de ces lésions posent un défi constant au chirurgien quant au choix du traitement qui doit être adapté au type de lésion et à son siège anatomique surtout en situation d'urgence (Arvieux, 2009).

$\mathrm{Au}$ Togo, nous notons depuis quelques années une augmentation du trafic routier, et de ce fait une augmentation des accidents de circulation qui sont à l'origine de traumatismes graves. Ainsi des lésions traumatiques graves de l'abdomen sont aussi observées et prise en charge en situation d'urgence avec un plateau technique relativement satisfaisant. Nous rapportons dans notre étude, deux cas de lésions traumatiques du cadre duodéno-pancréatique traitées chirurgicalement survenues dans les circonstances d'accidents de la voie publique en rappelant les circonstances diagnostiques et en décrivant la conduite thérapeutique et les suites opératoires dans nos conditions d'exercice au CHU Sylvanus Olympio de Lomé au Togo.

\section{Patients et méthode}

Nous avons mené une étude prospective sur une période de 12 mois dans le service des urgences chirurgicales du CHU SO de Lomé (Togo), d'Aout 2018 à Juillet 2019. Elle a intéressé 2 patients, un de sexe féminin et l'autre de sexe masculin, tous jeunes âgés respectivement de 18 et 27 ans. Les patients inclus étaient pris en charge pour des lésions traumatiques du cadre duodéno-pancréatique traitées chirurgicalement survenues dans les circonstances d'accidents de la voie publique. Ces patients étaient admis aux urgences chirurgicales prise en charge d'un polytraumatisme avec impact moto-piéton et moto-auto.

\section{Première observation}

Il s'agit d'un sujet de 27 ans, capable majeur de sexe masculin, adressé pour la prise en charge d'un poly traumatisme associant un traumatisme crânio-encéphalique et crânio-facial avec perte de connaissance initiale, et un 
traumatisme fermé de l'abdomen survenus par accident de la voie public impact moto-auto il y a 24 heures. Dans les circonstances, le patient était à moto, sans porter de casque, lorsqu'il a heurté un camion en stationnement ; le mécanisme exact est imprécis. Il y avait une notion de consommation d'alcool. Il n'y avait pas de fièvre ni de trouble du transit. Il a donc été conduit au Centre Hospitalier Régional de Dapaong puis au CHU SO pour une meilleure prise en charge. Il n'a pas d'antécédents pathologiques particuliers, ni d'allergie connue. L'examen clinique à l'admission permettait de noter une température normale à $36^{\circ} 4 \mathrm{C}$, un état général conservé avec un état hémodynamique stable, une conscience normale avec un score de Glasgow à 15/15, une rhinorragie associée à des plaies palpébrales suturées et une limitation des mouvements oculaires en haut et en dehors à l'œil droit. L'abdomen était de morphologie relativement normale, rétracté avec un point d'impact para ombilicale gauche à type d'une plaie superficielle linéaire d'environ $3 \mathrm{~cm}$; il était peu sensible dans l'ensemble sans cicatrice d'une laparotomie antérieure; le toucher pelvien était indolore. L'alcoolémie n'avait pas été dosée. Une tomodensitométrie cérébrale réalisée notait une fracture effondrement du rebord orbitaire supérieur droit et une contusion oedématohémorragique frontale droit. Un traitement antalgique, anxiolytique et une surveillance neurologique avait été institué. Quarante huit heures après, sont apparues des douleurs abdominales généralisées sans notion d'arrêt du transit ; un nouvel examen clinique notait une sensibilité abdominale généralisée et une défense diffuse, une matité modérée des flancs et une auscultation abdominale normale; le un toucher pelvien notait une sensibilité et un bombement du cul-de-sac de Douglas. Le bilan paraclinique notait une hyperleucocytose à 12400/mm3 et un taux d'hémoglobine à $13,2 \mathrm{~g} / \mathrm{dl}$ et un pneumopéritoine sans niveau hydroaérique à la radiographie de l'abdomen sans préparation (Figure 1). Le diagnostic d'une péritonite par perforation d'un organe creux a été posé et des mesures de réanimation institué avec une antibiothérapie à large spectre fait d'une céphalosporine de $3{ }^{\text {ème }}$ génération à type de ceftriaxone et d'un imidazolé à type de métronidazole. Une laparotomie exploratrice avait été décidée et réalisée ; ce qui à permis de découvrir un hémopéritoine d'environ $1000 \mathrm{ml}$ avec une plaie du grand épiploon recouvert de gros caillots; la mobilisation du colon transverse permettait de découvrir une infiltration d'une collection bilio-gazeuse associée à des taches de lyse graisseuse du fascia de Toldt et de la racine du mésocolon transverse ; le décollement du fascia de Toldt notait une cellulite retropéritonéale sur une perforation linéaire contuse de $3 \mathrm{~cm}$ à la face antérieure de duodénum à D4 et une infiltration œéémateuse du corps du pancréas sans rupture évidente du parenchyme pancréatique (figure 2). Le reste de l'exploration de la cavité péritonéale était normale. Comme geste, il était réalisé une aspiration liquide sanglant et les caillots, une excision des 
berges contuses de la perforation et une suture en point séparé au fil lentement résorbable 3/0 de celle-ci, un lavage minutieux et un drainage large par deux lames ondulés laissées à proximité du duodénum. Les suites opératoires ont été simples, avec une reprise du transit à $\mathrm{J} 3$, une réalimentation à $\mathrm{J} 5$ avec ablation de la lame de Delbet à $\mathrm{J} 5$ et une sortie du patient à J12. Un transit baryté réalisé trois mois après l'intervention a montré une reperméabilisation de l'ensemble du cadre duodénal.

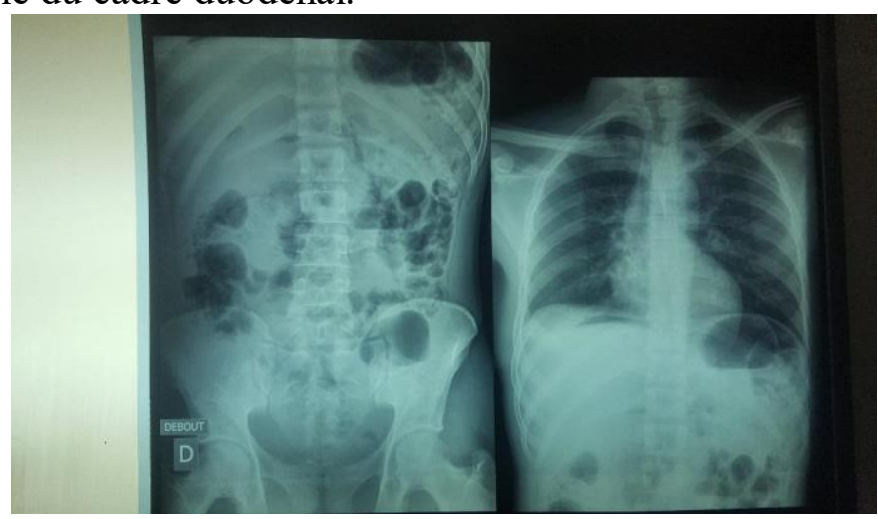

Figure 1: image de radiographie de l'abdomen sans préparation mettant en évidence un croissant gazeux (un pneumopéritoine) avec un niveau hydroaérique de siège épigastrique.

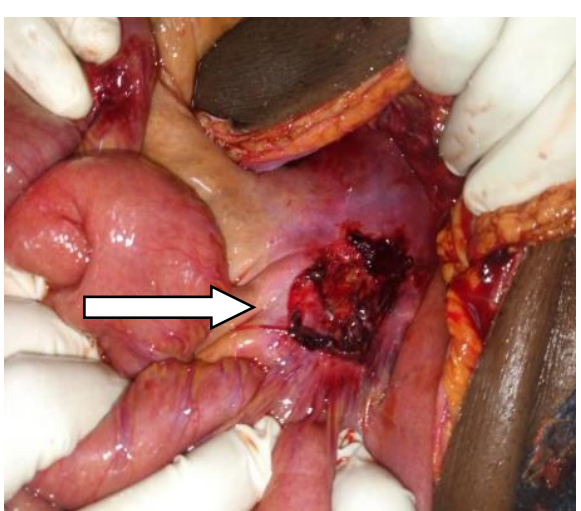

$2 \mathrm{~A}$

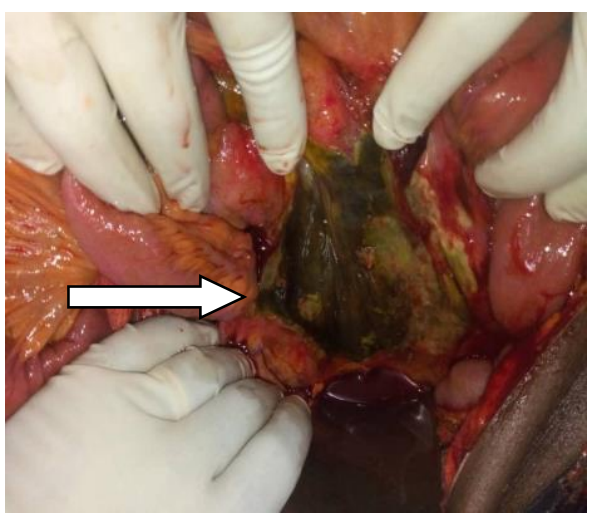

2B

Figure 2:

$2 \mathrm{~A}=$ Plaie contuse de racine du mésotransverse.

$2 \mathrm{~B}=$ une infiltration d'une collection bilio-gazeuse associée à des taches de lyse graisseuse du fascia de Toldt et de la racine du mésocolon transverse ; le décollement du fascia de Toldt notait une cellulite retropéritonéale.

\section{Deuxième observation}

Il s'agissait d'une jeune fille de 18 ans, capable majeur, admise pour un polytraumatisme associant un traumatisme crânio-encéphalique avec perte de connaissance de durée indéterminée et un traumatisme ferme de l'abdominal. Le polytraumatisme était survenu environ 72 heures avant son 
admission au CHU SO dans le cadre d'un accident de la voie public impact moto-piéton. La patiente marchait sur le rez-de-chaussée lorsqu'elle avait été heurtée par un motocycliste ; le mécanisme exact est imprécis. Il s'en était suivi un traumatisme crânio-encéphalique avec perte de connaissance et des plaies superficielles sur le corps; la patiente avait été conduite dans une clinique de la place où un traitement comportant des antalgiques, antiinflammatoires, des soins locaux des plaies et une surveillance neurologique avaient été institués. Les suites de cette prise en charge avaient été simples et le retour à domicile de la patiente avait été autorisé après 24 heures de surveillance. Trois (03) jours après la survenue de l'accident, des douleurs abdominales atroces diffuses d'intensité croissante étaient apparues, associées à une fièvre, à des nausées et vomissements, et un arrêt du transit ; ce qui avait motivé un transfert au CHU SO pour une meilleure prise en charge. La patiente n'a pas d'antécédents pathologiques particuliers, ni d'allergie connue.

L'examen clinique à l'admission notait une patiente consciente mais asthénique, très algique, présentant une pâleur palmo-plantaire sévère, une stabilité hémodynamique, un abdomen distendu sans point d'impact évident ni de cicatrice de laparotomie, très douloureux dans son ensemble avec une défense généralisée, une matité des flancs et un silence abdominal ; le toucher pelvien notait une douleur avec bombement du cul-de-sac de Douglas. Nous avons institué un traitement antalgique et antibiotique, des mesures de réanimation et de surveillance. Le bilan biologique réalisé notait une leucocytose à $6770 \mathrm{~GB} / \mathrm{mm} 3$, une anémie sévère avec un taux d'hémoglobine à $05,8 \mathrm{~g} / \mathrm{dl}$. La radiographie de l'abdomen sans préparation avait montré une stase stercorale importante sans pneumopéritoine, ni de niveau hydroaérique (Figure 3). Une échographie abdominale notait un épanchement liquidien de grande abondance associé à une rupture de la rate et une infiltration du cadre pancréatique. Le diagnostic d'une péritonite aigue généralisée était posé et des mesures de réanimation instituées associant une transfusion sanguine, un traitement antalgique et une antibiothérapie à large spectre fait d'une céphalosporine de $3^{\text {ème }}$ génération à type de ceftriaxone et d'un imidazolé à type de métronidazole. Une laparotomie exploratrice avait été indiquée et réalisée en urgence, ce qui avait permis de noter l'issue d'un liquide hématique d'environ $1200 \mathrm{cc}$ avec des taches de lyse graisseuse de l'épiploon, une fracture comminutive de la rate avec atteinte du pédicule hilaire siège d'un saignement en nappe, une infiltration œdémateuse du pancréas sans rupture évidente du parenchyme pancréatique donc sans atteinte du canal de Wirsung et sans lésion du cadre duodénal (figure 4). Nous réalisons une splénectomie totale sous contrôle vasculaire puis procédé au lavage abondant minutieux de la cavité péritonéale, à la mise en place de deux lames ondulées à proximité du pancréas et dans l'hypochondre droit. Les suites opératoires ont été marquées par une fièvre qui résolue progressivement sous traitement, avec la 
reprise du transit à $\mathrm{J} 3$, la reprise d'une alimentation à $\mathrm{J} 6$ et la sortie du patient à $\mathrm{J} 21$. Une TDM abdominale de contrôle avait été demandé mais n'a pu être réalisée faute de moyens financiers. Les glycémies réalisée en postopératoires étaient normales.

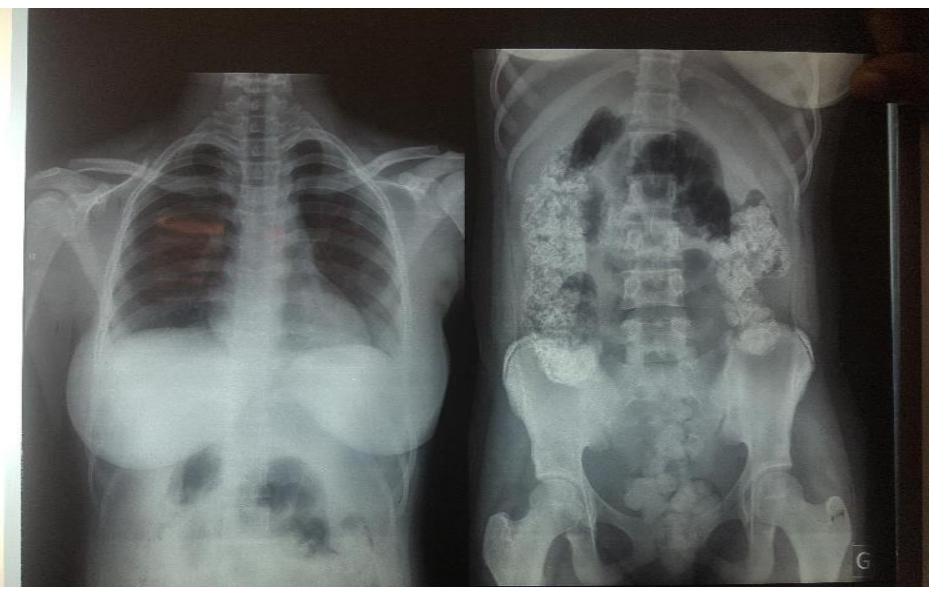

Figure 3 : image de radiographie de l'abdomen sans préparation mettant en évidence une stase stercorale colique sans pneumopéritoine ni de niveaux hydroaériques.

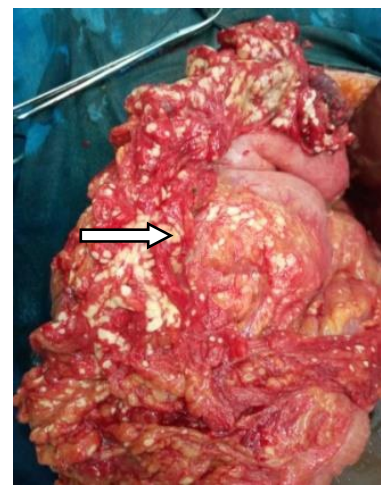

$4 \mathrm{~A}$

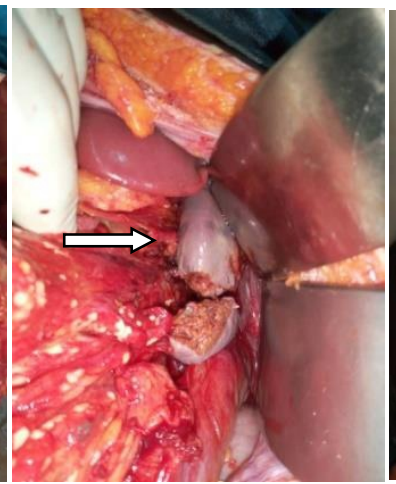

4B

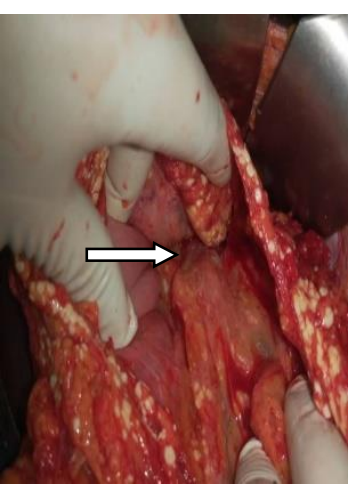

$4 \mathrm{C}$

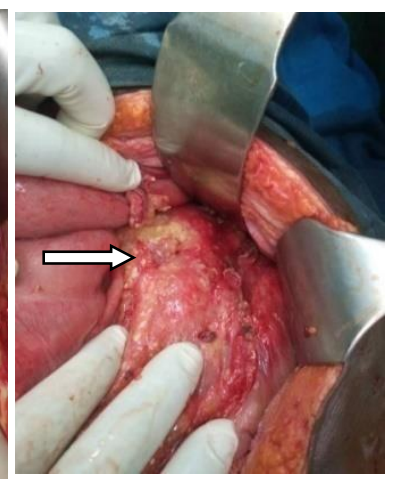

4D

Figure 4 :

$A=$ aspect de l'étage sus-mésocolique après aspiration de l'hémopéritoine

$\mathrm{B}=$ fracture la rate

$\mathrm{C}=$ ouverture le l'arrière cavité des épiploons

$\mathrm{D}=$ infiltration du pancréas après splénectomie

\section{Discussion}

Les lésions traumatiques fermées du pancréas et du duodénum sont rares par rapport aux autres lésions viscérales lors des traumatismes abdominaux (Farrell, 1996 ; Beyrouti, 2005 ; Arvieux, 2009). Ceci s'explique par la situation retro péritonéale profonde de ces organes qui leur garantie une protection lors des traumatismes. Et donc toute atteinte de ces organes sousentend un traumatisme violent. Considérant les circonstances de survenues des 
traumatismes dans notre étude, les contextes étaient tous des accidents de la voie publique survenus la nuit et les impacts étaient moto-piéton et moto-auto avec l'automobile en stationnement; ce qui témoigne de la violence des traumatismes, surtout qu'ils surviennent dans un contexte de route non éclairée la nuit donc une visibilité réduite, un contexte de non respect du code et aussi de conduite dans un état d'ivresse des conducteurs.

La fréquence des traumatismes duodéno-pancréatiques (TDP) est en augmentation (Beyrouti, 2005). Les plus grandes expériences émanent des 'Trauma centers' américains (Asensio, 1993). Leur fréquence est estimée dans la littérature entre 0,5 et $15 \%$ des traumatismes abdominaux (Carrel, 1990 ; Errougani, 1997 ; Perissat, 1991). Lors d'une revue de la littérature, ASENSIO et collaborateurs (Asensio, 2002), a estimé l'incidence des lésions pancréatiques entre 0,2 et $6 \%$, alors que celle des lésions duodénales est comprise entre 3 et $5 \%$ des traumatismes abdominaux. Dans notre série, la fréquence des TDP était de $2,3 \%$ des traumatismes abdominaux.

L'étiologie des TDP est dominée par les accidents de la voie publique qui constituent d'ailleurs la première cause (Beyrouti, 2005). Mais il existe aussi d'autres causes caractérisées par la recrudescence des agressions qu'elles soient secondaires à des armes blanches ou à des armes à feu ; et dans les pays développés l'amélioration des mesures de réanimation et des conditions de ramassage permet d'amener au bloc opératoire des blessés porteurs de lésions jusqu'alors mortelles sur le terrain d'accident (Asensio, 1993 ; Carrel, 1990 ; Errougani, 1997). Par contre pour Buenaventura (Buenaventura, 2007) et Gonzalo et collaborateurs (Gonzalo, 2015), les traumatismes abdominaux fermés survenant lors de festivals équestres populaires dans leur région constituent un risque élevé de traumatisme pancréatique.

Les traumatismes duodénopancréatiques rentrent dans le cadre des contusions abdominales à haute énergie et impliquent un écrasement les structures rétropéritonéales contre la colonne vertébrale (Jurkovich, 2013). La localisation rétropéritonéale du pancréas signifie que les signes et les symptômes provoqués par la lésion sont non spécifiques et difficiles à objectiver (Jurkovich, 2013 ; Arvieux, 2009). C'est ainsi que dans notre étude, l'expression symptomatique des lésions duodénopancréatiques objectivées étaient survenue 3 jours après l'accident et aucun symptôme initialement ne permettait de penser à l'atteinte de ces organes; et il s'agissait dans tous les cas d'un abdomen aigu chirurgical. Beyrouti et collaborateurs (Beyrouti, 2005) notait que le traumatisme est apparu par un abdomen chirurgical urgent chez 9 patients, secondairement dans 3 cas, tardivement dans 2 cas. Il en ressort une absence réelle de parallélisme entre l'expression clinique et l'aspect anatomoclinique d'une atteinte du cadre duodénopancréatique. L'expression clinique initiale varie de l'absence de tout signe clinique initial à un tableau péritonéal franc; et dans le cas d'une atteinte pancréatique, 
malheureusement il n'est pas possible de préjuger d'une atteinte canalaire en fonction de la symptomatologie (Bradley, 1998 ; Jurkovich, 2013 ; Arvieux, 2009). Il y a lieux d'insister sur l'intérêt de l'usage des protocoles Advanced Trauma Life Support dans les salles d'urgence (Subramanian, 2007 ; American, 2008 ; Asensio, 2003) qui doit orienter les praticiens vers les examens complémentaires initiaux utiles en urgence. Les preuves suggérant une lésion pancréatique potentielle incluent des antécédents de traumatisme à haute énergie dans l'épigastre et aussi des agressions avec atteinte épigastrique (Arvieux, 2009). En ce qui concerne les examens complémentaires, avec certaines limitations, le scanner est le test d'imagerie de choix pour le diagnostic et la stadification en phase aiguë ((Arvieux, 2009, Maeda, 2013).

Dans notre étude, le scanner n'était pas disponible en urgence dans notre structure hospitalière et sa réalisation dans les structures privées implique des moyens financiers énormes par rapport au niveau socioéconomique des blessés; dans ces cas on optait pour une laparotomie exploratrice urgence. Rappelons que si la suspicion de lésion pancréatique persiste et que les résultats de la tomodensitométrie sont négatifs, une cholangiopancréatographie rétrograde endoscopique (CPRE) et / ou une cholangiopancréatographie par résonance magnétique CPRM peuvent s'avérer utiles. La CPRE est la meilleure technique d'imagerie pour visualiser le Wirsung, mais sa disponibilité est limitée (Jurkovich, 2013). Dans notre série, nous ne disposons pas de ces moyens d'exploration paraclinique en urgence et le bilan lésionnel précis est réalisé en peropératoire dans le cadre d'un abdomen aigu chirurgical. Ce qui concernait la lésion duodénale, nous avions réalisé une excision des berges de la perforation et procédé à la fermeture par des points séparées au fil résorbable. Concernant les lésions pancréatiques, il est utile de les gérer conformément à l'Association américaine pour la chirurgie des traumatismes (Maeda, 2013). La lésion du pancréas à type d'une section complète (types III - V) est une indication de traitement chirurgical (Subramanian, 2007). Dans tous les cas de notre étude, aucun examen complémentaire préopératoire n'était réalisé pour apprécier l'atteinte du canal de Wirsung et le geste conservateur du parenchyme pancréatique était le choix thérapeutique privilégié avec un large drainage de la région duodénopancréatique. La procédure chirurgicale la plus étendue pour une section pancréatique traumatique complète de l'isthme est la résection du corps, de la queue et de la rate du pancréas. En moyenne, la pancréatectomie distale implique une résection de $56 \%$ de la glande pancréatique (Hutchins, 2002 ; Jones 1990). Dans notre étude, le cas de la contusion pancréatique associant une fracture de la rate avait bénéficié d'une splénectomie totale ; ce d'autant plus qu'aucune solution de continuité n'avait été objectivé dans le parenchyme pancréatique. Bien que l'insuffisance endocrine et / ou exocrine du pancréas soit rare après un traumatisme 
pancréatique si plus de $20 \%$ du tissu glandulaire est conservée, il est d'autant plus important de conserver autant de tissu pancréatique et de rate que possible chez les patients jeunes (Jurkovich, 2013 ; Jacko, 2012). Dans le cas de la préservation du pancréas distal, il est courant de pratiquer une anastomose pancréato-jéjunale et un Roux Y (Beyrouti, 2005 ; Arvieux, 2009).

\section{Conclusion}

Les lésions traumatiques du cadre duodéno-pancréatique sont rares et rentrent souvent dans le cadre de polytraumatisme grave. Leur expression clinique est non spécifique et l'abdomen chirurgical aigu est leur mode de révélation. L'usage des protocoles Advanced Trauma Life Support dans les salles d'urgence est donc d'une importance capitale dans la prise en charge des polytraumatisés.

\section{References:}

1. Farrell RJ, Krige JE, Bornman PC et al. Operative strategies in pancreatic trauma. Br J Surg 1996, 83: 934-7.

2. Beyrouti MI, Beyrouti R, Kchaou I, Gharbi W, Elleuch S, Tafech I, Frikha F, Ben Amar M, Ghorbel A, Bouaziz M, Karoui A, Sellami A. Duodeno-pancreatic trauma. About 14 cases. Tunis Med. 2005 Feb;83(2):73-82.

3. Feliciano DV, Martin TD, Cruse PA et al. Management of combined pancreatoduodenal injuries. Ann Surg 1987, 205 (6): 673-80.

4. Bradley EL, 3RD, Young PR, Jr., Chang MC et al. Diagnosis and initial management of blunt pancreatic trauma: guidelines from a multiinstitutional review. Ann Surg 1998, 227 (6): 861-9

5. Jurkovich G.J. Pancreatic and duodenal injuries. In: Cassan A., editor. vol. 1. B.C. Decker; Spanish Ed. Barcelona: 2013. (ACS Surgery Principles \& Practice). pp.21-35.

6. Catherine Arvieux, David Voirin, Christian Létoublon. Chirurgie des traumatismes du pancréas. Elsevier Masson, édition 2009 : 102-104.

7. Asensio J. A., Feliciano D. V., Britt L. D., Kerstein M. D. Management of duodenal injuries. Current problems in surgery 1993; 30 (11): 10211100.

8. Carrel T., Lerut J., Blumgart L. H. Diagnostic et traitement des lésions traumatiques du duodénum et du pancréas. Analyse de 21 cas. J Chir $1990 ; 127, \mathrm{n}^{\circ} 10: 438-444$.

9. Errougani A, Ameur A, Chkoff R, El Elaj A, Balafrej S. Les traumatismes duodéno-pancréatiques. A propos de 30 observations. J Chir $1997 ; 134, \mathrm{n}^{\circ} 1: 9-13$.

10. Perissat J., Collet D., Arnoux R., Salloum J., Bikandou G. Traumatismes du duodéno-pancréas. Principes de technique et de 
tactiques chirurgicales. Editions techniques- Encycl Med Chir., techniques chirurgicales, appareil digestif, 40898, 1991, 15 p.

11. Asensio J. A., Petrone P., Roldan G., Pak-Art R., Salim A. Pancreatic and duodenal injuries. Complex and lethal. Scandinavian Journal of Surgery 2002; $91: 81-86$.

12. Buenaventura A. Horse at the festival. In: Buenaventura A., editor. Menorca, Horses and Popular Tradition. Menorca Editorial; Mahon: 2007.

13. Gonzalo Martín Martín, Patricia Jiménez Morillas, José C. Rodríguez Pino, José M. Morón Canis, Francesc X. González Argenté. Reconstruction after pancreatic trauma by pancreaticogastrostomy. Int J Surg Case Rep. 2015; 9: 92-94.

14. Subramanian A., Dente C.J., Feliciano D.V. The management of pancreatic trauma in the modern era. Surg. Clin. N. Am. 2007;87:1515-1532.

15. American College of Surgeons . Advanced trauma life support. In: Scardiglia P.N., editor. Advanced Trauma Life Support. 8th edition. American College of Surgeons; Chicago: 2008.

16. Asensio J., García J.C., Petrone P., Roldán G., Pardo M. Pancreatic trauma: complex lesions, difficult treatments. Cir. Esp. 2003;74:124133.

17. Maeda K., Ono S., Baba K., Kawahara I. Management of blunt pancreatic trauma in children. Pediatr. Surg. Int. 2013;29:1019-1022.

18. Hutchins R., Hart R. Long-term results of distal pancreatectomy for chronic pancreatitis in 90 patients. Ann. Surg. 2002;236:6128.

19. Jones W., Finkelstein J. Managing pancreatic trauma. Infect. Surg. 1990;3:29-36.

20. Jacko S., Sim V., Cernero A., Todd S.R. Traumatic distal pancreatic transection: beware of the horses. J. Trauma Nurs. 2012;19:102-103. 